\title{
RELACIÓN ENTRE EL FITOPLANCTON Y LA SALINIDAD, DE LAS LAGUNAS INTRADUNARES DE LA DEHESA de la Albufera (Valencia)
}

\author{
Juan Miguel SORIA \& Manuela ROS
}

RESUMEN:Las áreas deprimidas entre los cordones dunares de la Dehesa de la Albufera se conocen con el nombre popular de «malladas». Algunas de ellas se inundan formando pequeñas lagunas de profundidad escasa (entre 0.5 y $1.9 \mathrm{~m}$ ) y de conductividad variable. Mientras las más profundas son permanentes, las someras son temporales, permaneciendo inundadas sólo las estaciones húmedas. El fitoplancton de estas lagunas se distribuye relacionado principalmente con esta salinidad variable y también influido por la inundación provocada con aguas provenientes de la Albufera, que ocasiona la aparición de especies propias del lago hasta que son sustituidas por las propias que corresponde.

Palabras clave: Fitoplancton, Salinidad, Lagunas costeras.

ABSTRACT: The flooded areas between the dunes in the «Dehesa de La Albufera» are known as «mallades». They are lagoons of limited depth (between 0.5 and $1.9 \mathrm{~m}$ ) and variable conductivity. While the deepest lagoons are permanent, the shallowest are temporary. Phytoplankton is widely distributed and relationed with salinity and chloride concentration, except when a temporary lagoon, named Rincón 3, is inundated with Albufera's water. Then, all phytoplankton that appears is the same of Albufera, and is replaced some weeks after.

Key words: Coastal lagoons, Phytoplankton, Salinity.

\section{INTRODUCCIÓN}

La Dehesa es una barra arenosa que se extiende paralela a la costa y separa el Mar Mediterráneo de la Albufera de Valencia. Tiene unos $14 \mathrm{~km}$ de longitud y una anchura variable, entre 500 y $1000 \mathrm{~m}$. Fue formada porque un golfo marino creado por un hundimiento, se fue rellenando por aluviones cuaternarios con un fondo de gravas y limos. Las aportaciones de materiales fluviales es fundamental en el proceso de 
relleno de la zona deprimida, formándose una restinga litoral. En la conformación final ha desempeñado un papel importante la corriente longitudinal marina de deriva, que es oblicua a la costa y terminó por cerrar los golfos primitivos (Ruiz, 1973).

La morfología primitiva ha sido muy alterada, y en la Dehesa se puede comprobar la presencia de grandes conjuntos dunares. El conjunto exterior, formado por grandes dunas con vegetación, que alcanzaban una altura entre 4 y $9 \mathrm{~m}$. El conjunto interior es de menor altura (unos $3 \mathrm{~m}$ ), pero de mayor anchura. Entre ellos se extiende longitudinalmente una amplia zona deprimida de anchura variable y de difícil drenaje por donde circulaba el camino central de la Dehesa (Ayto. Valencia, 1980). Esta zona se conoce con el nombre de «Mallada», y designa por extensión a las lagunas intradunares temporales o permanentes, rodeadas de vegetación acuática, que la inundan y que se forman por la proximidad del nivel freático y las lluvias.

Las Malladas de la Dehesa conocidas como Garrofera, Larga y la Mata, tienen una forma aproximadamente longitudinal, por encontrarse limitadas por las dos dunas de la Dehesa, en dirección N-S. Mantienen el nivel del agua todo el año. Las Malladas denominadas Rincón 1, Rincón 2 y Rincón 3 se encuentran dentro del área de protección del «Racó de L'Olla» (en castellano: Rincón de la Olla), antiguo hipódromo. La figura 1 nos muestra la situación de estas lagunas con respecto a la Albufera y el Mar Mediterráneo. Se caracterizan por ser temporales, perdiendo el agua durante la estación seca. Rincón 3 se encuentra comunicada con las aguas de la Albufera por un canal, que permite la entrada o posible salida de las aguas desde o hacia la Albufera.

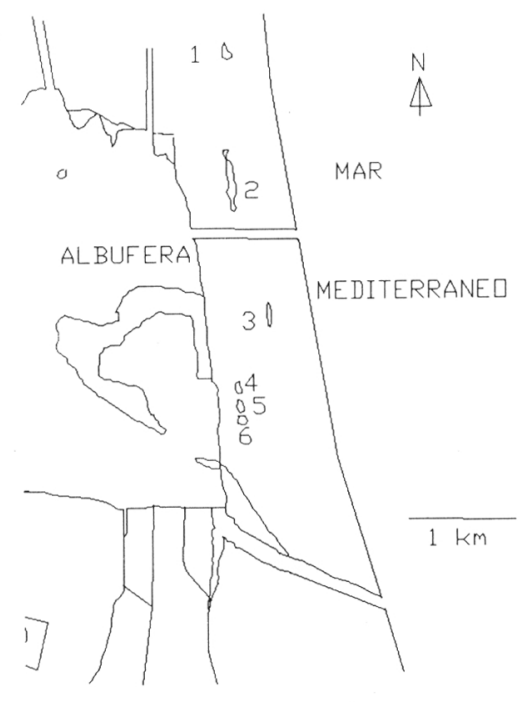

Figura 1. Situación de las lagunas intradunares en la Dehesa del Saler. Clave: 1, Garrofera. 2, Larga. 3, Mata. 4, Rincón 1.

5, Rincón 2. 6, Rincón 3.

\section{MÉTODOS}

Las tomas de muestras se realizaron desde febrero de 1987 hasta enero de 1988 , intentando que la frecuencia fuera de seis semanas aproximadamente.

Se tomaron muestras de agua a 0.2 $0.5 \mathrm{~m}$ de profundidad con botellas de $11 \mathrm{de}$ capacidad. De esta muestra se separaron diferentes fracciones, conservadas según la metodología descrita por Stainton et al (1977). Se conservó 11 aproximadamente de muestra fijada con lugol para la observación y recuento de fitoplancton, que se efectuaba dejando sedimentar en cubetas cilíndricas volúmenes de agua de 1 a $3 \mathrm{ml}$ durante 24 horas al menos (Utermohl, 1958). Posteriormente se observaba a 1000 
aumentos en microscopio invertido. La identificación de las especies dudosas se ha realizado en un microscopio normal a 2000 aumentos, tanto en campo claro como en contraste de fases. Para la clasificación de las algas se ha seguido a Desikachary (1959) para el grupo de las Cianobacterias; a Germain (1981) para las Diatomeas, y a HuberPestalozzi (1938, 1955, 1961, 1968, 1971, 1978) para los demás grupos fitoplanctónicos.

Se calculó el índice de diversidad según Shannon-Wiever (Legendre, 1979), que nos da una medida de la organización del sistema. Se emplearon para el proceso estadístico los programas BMDP 1 D, que nos muestra la variabilidad de los parámetros físicoquímicos y su distribución en las Lagunas, y $4 \mathrm{M}$ que realiza un análisis multivariante de las variables normalizadas. Para ello se consideraron los valores de la transformación logarítmica de las variables originales. Se obtuvo la matriz de correlaciones entre variables y una agrupación «Cluster» de ellas (Sneath y Sokal, 1973).

\section{RESULTADOS}

\section{MINERALIZACION DE LAS AGUAS}

Las aguas de las lagunas tienen escaso contenido en nitrógeno y fósforo (Tabla 1), y no parecen presentar problemas de contaminación por vertidos. La alcalinidad es elevada y los valores del cloruro y sulfato son variables según la salinidad propia de cada charca. La concentración de sulfato es baja en general, entre 0.1 y $2.5 \mathrm{~g} / 1$, mientras que la del cloruro presenta los valores más variables. Se califican las aguas salobres en aguas oligohalinas cuando tienen hasta $2 \mathrm{~g} / 1$ de cloruro, mesohalinas de 2 a $10 \mathrm{~g} / 1$, polihalinas de 10 a $17 \mathrm{~g} / 1$, y aguas hiperhalinas cuando éstas se evaporan, y aumenta más la concentración de sales (Margalef,1983).

Estas lagunas, pese a su proximidad no son homogéneas, y son una muestra de la importancia que tiene conocer la distribución de las masas del acuífero de la Dehesa: la primera, Rincón 1, es oligohalina, Rincón 2 es mesohalina con tendencia polihalina (entre 3 y $14 \mathrm{~g} / 1$ de cloruro) y Rincón 3 es polihalina llegando a hiperhalina cuando las aguas están en niveles bajos. Esta última laguna es el centro de la futura reserva biológica del «Racó de L'Olla», y se ha construido un canal que la enlaza con la Albufera, con la intención de inundarla en momentos de bajo nivel freático, o desaguarla en momentos de nivel elevado. Así, durante las lluvias del invierno de 1986-87, el acuífero de la Dehesa se recargó de tal manera que en lugar de entrar agua desde el lago, salía hacia él. El agua que fluía desde la depresión era salobre, con un contenido de $12 \mathrm{~g} / 1$ de sales disueltas. Cuando bajó el nivel, aumentó la concentración hasta $60 \mathrm{~g} / 1$ depositándose las evaporitas. Posteriormente, en agosto de 1987 se inundó con aguas de La Albufera (salinidad en torno a $1 \mathrm{~g} / \mathrm{l}$ ) que disolvieron los precipitados y salinizaron el agua, muy concentrada al principio, y menos después de las tormentas de otoño de 1987 , cuando bajó hasta $5 \mathrm{~g} / 1$ por dilución con aguas de lluvia (Soria \& al, 1988) 


\begin{tabular}{|c|c|c|c|c|c|c|c|c|c|c|c|c|}
\hline & \multicolumn{2}{|c|}{ Garrofera } & \multicolumn{2}{|c|}{ Larga } & \multicolumn{2}{|c|}{ Mata } & \multicolumn{2}{|c|}{ Rincón 1} & \multicolumn{2}{|c|}{ Rincón 2} & \multicolumn{2}{|c|}{ Rincón 3} \\
\hline & media & std & media & std & media & std & media & std & media & std & media & std \\
\hline emperatura $\left({ }^{(2} \mathrm{C}\right)$ & 21,0 & 7,1 & 19,0 & 6,1 & 18,4 & 6,4 & 13,9 & 3,7 & 15,3 & 3,4 & 21,1 & 8,2 \\
\hline Conductividad $(\mathrm{mS} / \mathrm{cm})$ & 2,10 & 0,32 & 24,71 & 9,25 & 1,61 & 0,51 & 2,91 & 0,79 & 18,41 & 11,44 & 36,57 & 25,40 \\
\hline Oxígeno (mg/l) & 7,49 & 2,80 & 5,31 & 2,78 & 4,66 & 2,38 & 4,95 & 2,66 & 12,90 & 5,16 & 11,77 & 5,44 \\
\hline $\mathrm{pH}$ & 7,92 & 0,55 & 7,82 & 0,57 & 7,69 & 0,72 & 7,75 & 0,23 & 8,37 & 0,49 & 8,64 & 0,92 \\
\hline Alcalinidad (meq/l) & 4,64 & 1,19 & 7,58 & 1,61 & 6,96 & 1,83 & 4,97 & 1,08 & 6,61 & 4,78 & 5,02 & 3,01 \\
\hline Clorur & 17,82 & 5,64 & 395,73 & 201,36 & 13,89 & 5,04 & 59,53 & 61,04 & 256,98 & 146,78 & 608,17 & 500,54 \\
\hline Sulfato (meq/l & 4,07 & 0,26 & 10,15 & 4,98 & 2,79 & 4,25 & 3,05 & 1,39 & 16,50 & 8,88 & 30,84 & 23,41 \\
\hline Nitrato (uM & 1,56 & 2,74 & 74 & 1,64 & 97 & 6,50 & 0,92 & 83 & 1,01 &, 98 & 1,07 & 1,06 \\
\hline Nitrito (UM) & 0,32 & 0,52 & 39 & 0,58 & 8 & 25 & 0,22 & 13 & 0,14 & 12 & 0,07 & 0,07 \\
\hline Amonio (UM) & 31,58 & 41,09 & 53,96 & 77,27 & 3, & 3,47 & 6,06 & 5,89 & 1,22 & 1,86 & 1,00 & 1,35 \\
\hline Fosfato (UM) & 0,44 & 0,74 & 0,16 & 0,18 & 0,11 & 0,08 & 4,93 & 2,74 & 0,21 & 0,17 & 0,33 & 0,26 \\
\hline Silicato (UM) & 53,07 & 50,34 & 204,25 & 109,89 & 270,88 & 144,06 & 88,54 & 48,30 & 101,63 & 77,83 & 128,72 & 79,85 \\
\hline Clorofila A (mg/m3) & 27,71 & 34,78 & 29,25 & 10,91 & 27,69 & 10,74 & 9,90 & 11,36 & 36,06 & 13,93 & 57,66 & 24,36 \\
\hline Fitoplancton (ind/ml) & 86781 & 60941 & 28944 & 45673 & 40408 & 47317 & 2238 & 1915 & 50833 & 50617 & 27578 & 15681 \\
\hline
\end{tabular}

Tabla 1. Valores medios (media) y desviación estándar (std) de las variables consideradas en el estudio de las lagunas intradunares de la Dehesa del El Saler (Valencia).

\section{FITOPLANCTON}

La distribución de las especies del fitoplancton encontradas en la lagunas de la Dehesa es particular para cada una de ellas. El promedio de especies oscila entre 28 especies en la Laguna de la Garrofera y 12 especies en Rincón 1 (figura 2). Las familias con mayor número de especies son Bacillariophyceae y Chlorophyta, seguido de Cyanophyceae.

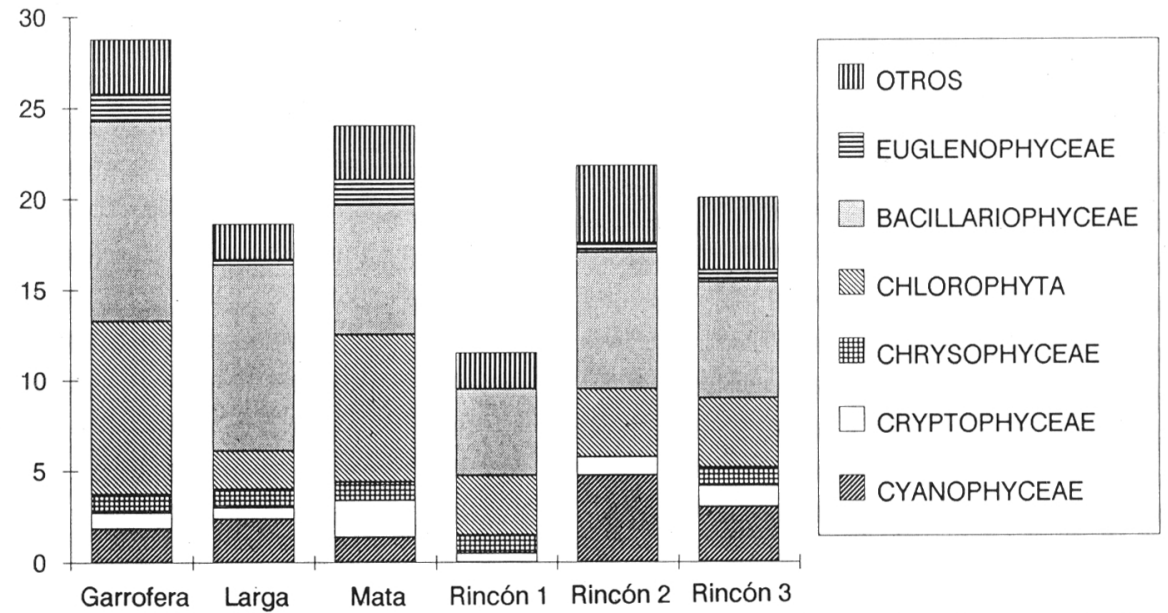

Figura 2. Valor medio del número de especies presentes en cada laguna intradunar de la Dehesa del Saler. 


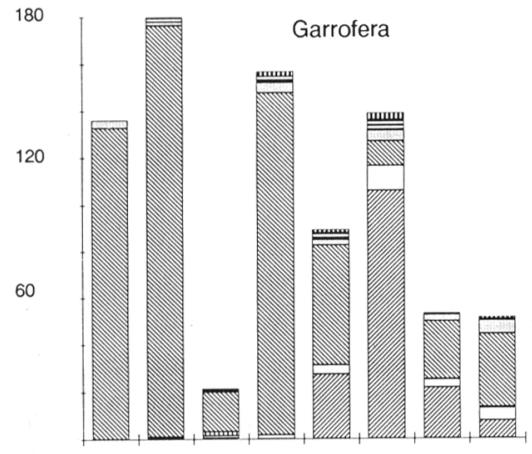

23-Feb8-Abr 26-May7-Jul 24-Ago14-Oct19-Nov12-En

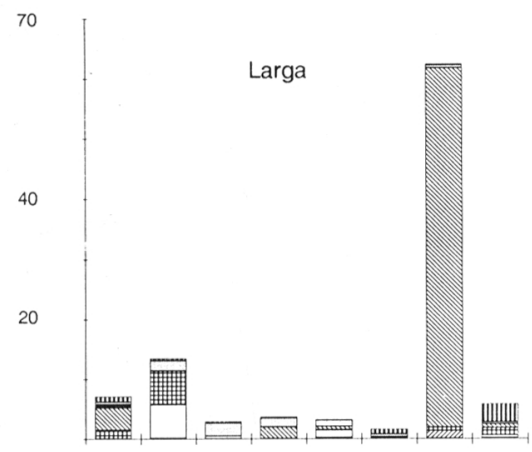

23-Feb8-Abr 26-May7-Jul 24-Ago14-Oct19-Nov2-En

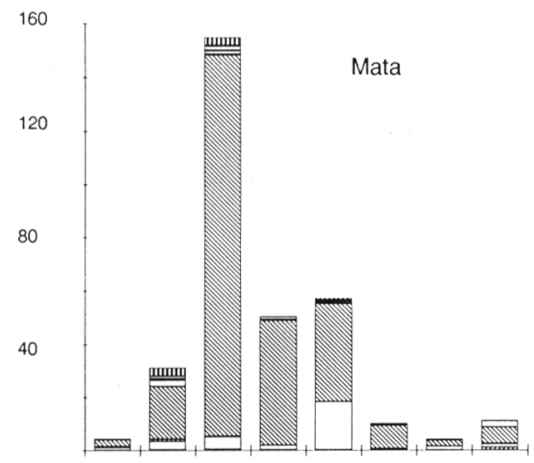

24-Feb8-Abr 26-May7-Jul 24-Ago14-Oct19-Nov12-En
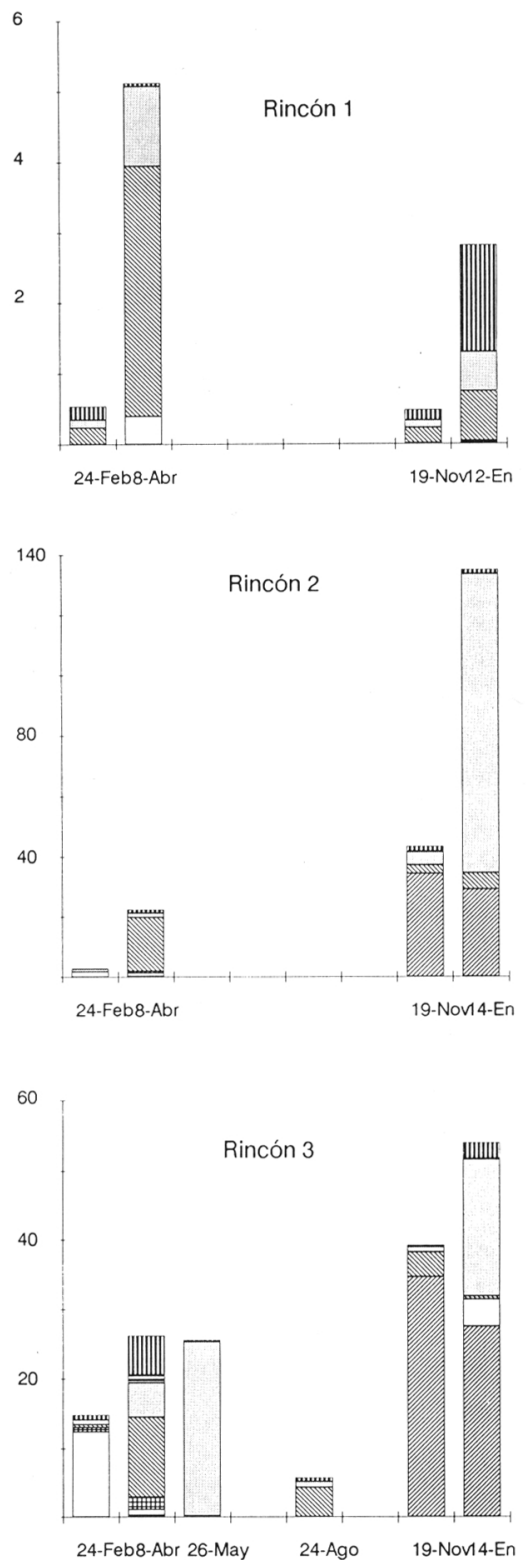

Figura 3. Representación del número de especies en cada muestreo en cada una de las lagunas de la Dehesa del Saler. El entramado corresponde a la simbología representada en la Fig. 2 (pág. 46). Valores expresados en miles de individuos por mililitro de muestra. 
Los valores medios para cada laguna del número de individuos por mililitro de muestra son variables (figura 3). Estos valores están comprendidos entre 86000 ind/ $\mathrm{ml}$ de media en la laguna de la Garrofera y 2300 ind/ml en Rincón 1. Por familias, las más abundantes son las Chlorophyta en las lagunas permanentes, mientras que las Bacillariophyceae y las Cyanophyceae son las más abundantes en las lagunas temporales.

En la Garrofera, dominan inicialmente las Chlorophyta, mientras que a finales del verano y otoño aparecen las Cyanophyceae, que vuelven a desaparecer en el invierno, aumentando de nuevo las primeras.

En la Larga aparece baja cantidad de individuos excepto en el otoño, tras la inundación de toda el área. Es un dominio de las Chlorophyta, especialmente de la especie Nephroselmis salina que no aparece más que en estos momentos.

En la laguna de la Mata son las Chlorophyta las dominantes en cualquier momento. Los máximos de población total se presentan en primavera y verano, y los mínimos en los momentos de mayor inundación.

Las lagunas del «Racó de L'Olla» presentan grandes fluctuaciones debidas sobre todo a sus características y a su temporalidad. Rincón 1 presenta una variabilidad entre las Chlorophyta en abril y flagelados de la familia Haptophyceae en enero. Se caracteriza sobre todo por la escasa densidad de población de individuos en cualquier momento. Rincón 2 presenta un crecimiento de Chaetoceros muelleri en enero, tras la inundación de otoño, y poblaciones importantes de Cyanophyceae provenientes de las aguas de la Albufera que llegan desde la laguna vecina Rincón 3. Esta última se caracteriza por tener su flora autóctona durante la primavera, formada inicalmente por Rhodomonas lacustris, sustituida luego por las Chlorophyta, Bacillariophyceae y Flagelados, y por último, en los momentos de mayor salinidad, la mayoría es de Nitzschia closterium. Antes de las lluvias se inundó con agua de la Albufera, y aparece una población en otoño e invierno formada por especies de Cyanophyceae propias de allí, como Oscillatoria aghardii, Oscillatoria redekei y en enero también aparece Chaetoceros muelleri, como en Rincón 2, ya comentado anteriormente.

Los valores bajos del índice de diversidad de Shannon indican inestabilidad en el sistema, mientras que las diversidades altas mayor organización. Presentan mayor diversidad en conjunto las lagunas permanentes que las temporales. Dentro de ellas, las de agua oligohalina aún tienen mayor diversidad que las polihalinas. Los valores máximos son de 3.9, siendo el valor medio de 2.6. Las lagunas más salobres tienen una diversidad media de 2 .

\section{CORRELACIONES}

Un análisis de correlación multivariante de las especies fitoplanctónicas y los parámetros físicoquímicos pone de manifiesto la relación entre ellos y las diferentes especies. Los grupos de correlación nos indican cómo las especies se distribuyen en función de la temperatura, salinidad y fosfatos. Para el conjunto de las lagunas, se han establecido los siguientes grupos de correlación: 
Un primer grupo nos agrupa aquellas especies presentes en las lagunas de mayor salinidad, tanto en la laguna Larga como en Rincón 3, y son Amphora lineolata, Synedra tabulata, Amphora coffeoeformis, Oxyrhis marina y Nitzschia closterium. Presentes sólo en la laguna Larga está Nephroselmis salina y Gymnodinium splendens. Exclusivamente en esta laguna, y en verano, se encuentran agrupadas las especies Chroococcus tenax, Gomphosphaeria aponina, Diploneis smithii, Plagiotropis lepidoptera, Amphora communata, Mastogloia lanceolata, Navicula pupula var. capitata y Nitzschia hungarica; es el momento en que alcanza los valores máximos en amonio y alcalinidad de las aguas, que aparecen muy correlacionados con estas especies. Por último, las especies Chaetoceros muelleri, Nitzschia closterium y otras indeterminadas del género Gymnodinium se presentan en los periodos de temperaturas bajas, otoño e invierno.

Un segundo grupo está formado por las especies presentes en las lagunas de aguas de conductividad más baja. Encontramos agrupadas las especies presentes sólo en la laguna de la Garrofera, como Merismopedia punctata, Tetraedron minimum, Navicula lanceolata, Crucigeniella tetrapedia, Nitzschia amphibia y Monoraphidium tortile. Asociadas a ellas están las especies presentes tanto en esta laguna como en la Mata. Son Cryptomonas erosa, Kirchneriella irregularis, Monoraphidium controtum, Didimocystis bicellularis, Synedra ulna Kirchneriella lunaris y Monoraphidium kormakovae.

El tercer grupo agrupa a las especies encontradas en las lagunas del Rincón 2 y Rincón 3, salobres, pero que se encuentran allí arrastradas por el flujo de agua procedente de La Albufera. Son Scenedesmus acuminatus, Oscillatoria aghardii, Lyngbya limnetica, Anabaenopsis circularis, Oscillatoria redekeii, Lyngbya contorta, Lyngbya bipunctata, Monoraphidium pusilum, Nitzschia palea var. tenuirostris y Nitzschia palea. Estas especies se encuentran normalmente allí (Soria et al, 1987) y aquí aparecen tras la inundación, y van siendo sustituidas por especies más propias de las aguas salobres.

\section{CONCLUSIONES}

Dos son los factores naturales que afectan a la distribución del fitoplancton en las lagunas intradunares de la Dehesa: por una parte la concentración de cloruro, y por otra la temporalidad de sus aguas. Un tercer factor es la manipulación humana inundándolas con aguas procedentes de otro ecosistema, que produce la aparición de especies alóctonas (en su mayoría Cianofíceas), que son sustituidas por las especies propias que corresponden a su calidad de aguas.

\section{AGRADECIMIENTOS}

Los autores agradecen a la Unidad Docente de Ecología del Departamento de Microbiología de la Fac. de Biológicas de la Universitat de Valencia su ayuda prestada en medios materiales; y a la Oficina Técnica Devesa-Albufera del Excmo. Ayto. de Valencia su apoyo económico en la realización del presente estudio. 


\section{BIBLIOGRAFÍA}

APHA, AWWA, WPCF-1985-Standard Methods for the examination of water and wastewater. American Public Health Association. 1268 págs.

AYUNTAMIENTO DE VALENCIA -1980- Estudios previos para la ordenación del monte de la Dehesa del Saler. Publ. Arch. Munic. Valencia. 95 págs.

DESIKACHARY, T.V. -1959-Cyanophyta. Indian Council of Agricultural Research. Nueva Delhi.

GERMAIN. H. -1981- Flore des Diatomées. Eds. Boubée. Paris. 441 págs.

HUBER-PESTALOZZI, G. -1938- Das phytoplankton des Susswassers. Allgemeiner Teil. Blaualgen. Bakterien. Pilze. E. Schweizerbart'sche Verlagsbuchhandlung (Erwin Nagele). Stuttgart. Band XVI. Teil. 1: 342 pp.

HUBER-PESTALOZZI, G. -1941- Das phytoplankton des Susswassers. Chrysophyceen. Farblose flagellaten. Heterokonten. (Idem). Teil. 2: 365 pp.

HUBER-PESTALOZZI, G. -1942- Das phytoplankton des Susswassers. Diatomeen. (Idem). Teil. 3: 549 pp.

HUBER-PESTALOZZI, G. 1955. Das phytoplankton des Susswassers. Euglenophyceae. (Idem). Teil. 4: 606 pp.

HUBER-PESTALOZI, G. -1961- Das phytoplankton des Susswassers. Chlorophyceae. Volvocales. (Idem). Teil. 5: 744 pp.

HUBER-PESTALOZZI, G. -1968- Das phytoplankton des Susswassers. Cryptophyceae. Choloromonadophyceae. Dinophyceae. (Idem). Teil. 6: 322 pp.

HUBER-PESTALOZI, G. -1972- Das phytoplankton des SOsswassers. Chlorophyceae. Tetrasporales. (Idem). Teil. 7: $116 \mathrm{pp}$.

LEGENDRE, L. \& P. LEGENDRE -1979- Ecologie numérique. Tome 2: La structure des dennées écologiques. Masson. Paris. 254 págs.

MARGALEF, R.-1983-Limnología. Omega. Barcelona. 1010 págs.

RUIZ, P. -1973- Monte de la Devesa. Gran Enciclopedia de la Región Valenciana, 4: 32.

SNEATH P.H.A. \& R.R. SOKAL -1973-Numerical Taxonomy. W.H. Foreman. San Francisco.

SORIA, J.M., W. COLOM \& L. BALLESTEROS -1988- Estudio comparado de la salinidad en «Les mallades de la Devesa», Valencia. Congreso Europeo de Ordenación del Territorio. Actas 1: 53-55.

SORIA, J.M., M. MIRACLE \& E. VICENTE - 1987- La Albufera de Valencia: Comunidades fitoplanctónicas en la campaña 1982-83. Vl Simp. Nac. Bot. Cript. Actas: 165- 172.

STAINTON, M.P., M.J. CAPEL \& F.A.J. ARMSTRONG -1977- The Chemical analysis of fresh water. Can. Fish. Mar. Serv. Misc. Spec. Publ. 25:180 p.

UTERMOHL, H. -1958- Zur Vervollkommung der quantitative Phytoplankton Methodik. Mitt. Int. Verein. Limnol., 9:1-38.

(Aceptado para su publicación en Junio de 1.990 )

Dirección de los autores: J.M. SORIA: Confederación Hidrográfica del Júcar. 46071 Valencia. M. ROS: Agencia del Medio Ambiente. Generalitat Valenciana. 